\title{
Strategies to Overcome Risks Associated with Endoscopic Biliary Stenting
}

\author{
Woo Hyun Paik and Yong-Tae Kim \\ Division of Gastroenterology, Department of Internal Medicine and Liver Research Institute, Seoul National University Hospital, Seoul \\ National University College of Medicine, Seoul, Korea
}

See "Risk Factors for Pancreatitis and Cholecystitis after Endoscopic Biliary Stenting in Patients with Malignant Extrahepatic Bile Duct Obstruction” by Ga Hee Kim, Si Kyong Ryoo, Jae Keun Park, et al., on page 598-605.

Transpapillary biliary stent placement with endoscopic retrograde cholangiopancreatography (ERCP) has been the initial treatment choice for the resolution of malignant extrahepatic bile duct obstruction. ${ }^{1}$ Endoscopic retrograde biliary drainage (ERBD) has advantages over percutaneous transhepatic biliary drainage in terms of physiology, patient comfort, maintenance of enterohepatic bile circulation, and having no external indwelling catheter requirement. However, ERBD has some drawbacks, including procedure-related pancreatitis and cholecystitis, which results from mechanical obstruction of the ampulla of Vater, pancreatic sphincter, or opening of the cystic duct by a biliary stent; hydrostatic injury caused by contrast dye injection; and chemical or allergic injury from the contrast dye. ${ }^{2}$ To prevent procedure-related pancreatitis and cholecystitis, knowledge and prevention of risk factors associated with these adverse events are important.

Kim et al. ${ }^{3}$ retrospectively analyzed risk factors for the aforementioned adverse events in 375 patients with unresectable extrahepatic malignant biliary obstruction. In this study,

Received: October 8, 2019 Revised: October 25, 2019

Accepted: October 28, 2019

Correspondence: Yong-Tae Kim

Division of Gastroenterology, Department of Internal Medicine and Liver Research Institute, Seoul National University Hospital, Seoul National University College of Medicine, 101 Daehak-ro, Jongno-gu, Seoul 03080, Korea

Tel: +82-2-2072-2944, Fax: +82-2-762-9662, E-mail: yongtkim@snu.ac.kr ORCID: https://orcid.org/0000-0002-4842-6874

(c) This is an Open Access article distributed under the terms of the Creative Commons Attribution Non-Commercial License (http://creativecommons.org/ licenses/by-nc/3.0) which permits unrestricted non-commercial use, distribution, and reproduction in any medium, provided the original work is properly cited. the incidence rates of procedure-related pancreatitis and cholecystitis were $26 \%$ and $8 \%$, respectively. Contrast dye injection in the pancreatic duct and metal stent placement were risk factors for post-ERBD pancreatitis, whereas histories of biliary drainage and plastic stent placement were protective factors for post-ERBD pancreatitis. In a previous study, contrast dye injection in the gallbladder and cystic duct invasion of the tumor are were factors for post-ERBD cholecystitis. It is interesting how the risk of cholecystitis, a well-known risk factor for post-ERBD cholecystitis, did not increase after metal stent insertion in this study. ${ }^{4}$ Selection bias may have influenced the results. In this study, ERCP was performed only by highly experienced operators who tend not to insert a metal stent when it can hinder biliary flow at the cystic duct orifice. However, the incidence of pancreatitis in the present study was higher than that reported in previous studies. The authors explained that the high incidence of pancreatitis was due to the routine check of serum amylase and lipase levels on the next day after ERCP. In addition to the above-mentioned reason, the incidence of pancreatitis might increase during selective cannulation of ERCP because the authors' hospital is a training institution where trainees are involved in initial cannulation. Unfortunately, in this study, the important technique-related factors for ERCP were missing from the analysis, including pancreatic sphincterotomy, difficult cannulation, and pancreatic deep wire cannulation. ${ }^{5}$

Some patient-related factors associated with adverse events of ERBD cannot be corrected; however, procedure-related factors can be manipulated by operators. The contrast agent 
should be carefully injected to avoid opacification of the pancreatic duct or gallbladder. Biliary metal stents may be carefully selected in patients with a high risk of post-ERCP pancreatitis or cholecystitis, such as younger and female patients and those with suspected sphincter of Oddi dysfunction and cystic duct invasion of the tumor. ${ }^{6,7}$ In the high-risk group of post-ERCP pancreatitis, prophylactic pancreatic stenting before or after biliary metal stent placement may be useful.

In this study, $42 \%$ of the patients with pancreatic cancer developed pancreatitis after ERBD, similar to the rate observed in controls. ERCP or ERBD does not increase the risk of post-procedure pancreatitis in patients with pancreatic cancer because the contrast dye cannot be injected across the stricture site of the pancreatic duct due to too tight stenosis. The authors did not mention the discrepancies between their results and those of previous studies. They also did not comment why cholangiocarcinoma was more frequent in the cholecystitis group (50\%) than in the non-cholecystitis group (18\%). This may be owing to the more frequent tumor involvement of the cystic duct orifice in cholangiocarcinoma. Although ERBD is the established modality for palliation of malignant biliary obstruction, it still carries risks of severe adverse events, including pancreatitis and cholecystitis. To overcome these risks, the operator must be aware of the risk factors and carefully perform ERBD.

Conflicts of Interest

The authors have no financial conflicts of interest.

ORCID

Woo Hyun Paik: https://orcid.org/0000-0001-8708-3280

\section{REFERENCES}

1. ASGE Technology Assessment Committee, Pfau PR, Pleskow DK, et al. Pancreatic and biliary stents. Gastrointest Endosc 2013;77:319-327.

2. Thaker AM, Mosko JD, Berzin TM. Post-endoscopic retrograde cholangiopancreatography pancreatitis. Gastroenterol Rep (Oxf) 2015;3:32-40.

3. Kim GH, Ryoo SK, Park JK, et al. Risk factors for pancreatitis and cholecystitis after endoscopic biliary stenting in patients with malignant extrahepatic bile duct obstruction. Clin Endosc 2019;52:598-605.

4. Jang S, Stevens T, Parsi M, et al. Association of covered metallic stents with cholecystitis and stent migration in malignant biliary stricture. Gastrointest Endosc 2018;87:1061-1070.

5. Freeman ML, DiSario JA, Nelson DB, et al. Risk factors for post-ERCP pancreatitis: a prospective, multicenter study. Gastrointest Endosc 2001;54:425-434.

6. Cao J, Peng C, Ding X, et al. Risk factors for post-ERCP cholecystitis: a single-center retrospective study. BMC Gastroenterol 2018;18:128.

7. Mine T, Morizane T, Kawaguchi Y, et al. Clinical practice guideline for post-ERCP pancreatitis. J Gastroenterol 2017;52:1013-1022. 